\title{
GPS + Galileo tightly combined RTK positioning for medium-to-long baselines based on partial ambiguity resolution
}

\author{
Guangcai Li ${ }^{1 *}$, Jianghui Geng ${ }^{1,2}$, Jiang Guo', Sen Zhou and Shuang Lin ${ }^{4}$
}

\begin{abstract}
With the modernization of the GNSS, the techniques of multi-GNSS navigation and positioning are becoming increasingly important. For multi-GNSS double-difference data processing, a tight combination (TC) strategy can provide more observations and higher reliability, which emploies a single reference satellite for all observations from different GNSS. However, multi-GNSS will bring some challenges to the high-dimension ambiguity resolution (AR). In this contribution, a GPS + Galileo tightly combined real-time kinematic (RTK) positioning strategy is proposed, which introduces the partial ambiguity resolution (PAR) method. A set of baselines ranging from about 22 to $110 \mathrm{~km}$ are used to test the positioning performance of this strategy. Experimental results demonstrate that the TC strategy can improve the success rate, but it can't increase the ambiguity ratio values. Using the PAR method can reduce convergence times and improve the ambiguity fixing rate. Combining the TC strategy with the PAR method can provide better positioning performance, especially for long baselines.
\end{abstract}

Keywords: GPS + Galileo, Inter-system double-differencing, Partial ambiguity resolution, Long-baseline

\section{Introduction}

With the modernization of Global Navigation Satellite System (GNSS), multi-GNSS navigation and positioning techniques are becoming increasingly important. Combining observations from various GNSS constellations significantly increases the number of observations and improves the positioning accuracy and reliability, especially in difficult environments ( $\mathrm{Li}$ et al., 2016a). Multi-GNSS double difference combination strategies include loose combination (LC) in which each of the systems uses its own reference satellite and no double differences are formed across systems (Zhang et al. 2003), and tight combination (TC) in which two systems use the same reference satellite and permitting double differences across different systems (Julien et al. 2003). Therefore, the TC strategy can provides more observations than the LC strategy. However, as the ambiguity dimension increases sharply, the success rate of integer ambiguity resolution is reduced (Teunissen et al., 1999), while the key requirement for real-time kinematic (RTK)

\footnotetext{
* Correspondence: guangcai.li@whu.edu.cn

'GNSS Research Center, Wuhan University, Wuhan, China

Full list of author information is available at the end of the article
}

is to quickly and correctly fix the ambiguities of carrier phase measurements. For multi-GNSS data processing, it is often impossible to fix all ambiguities simultaneously due to the large number of observations, which is even deteriorated in case of medium-to-long baselines (more than $20 \mathrm{~km}$ ) when various residual errors cannot be mitigated completely (Li et al., 2016, b).

To solve this problem, the idea of partial ambiguity resolution (PAR), which means to resolve a subset of the candidate ambiguities, was suggested to maintain a sufficiently high success rate (Teunissen et al., 1999). The selection of an ambiguity subset could be based on predefined subset sizes (Mowlam and Collier, 2004), ambiguity variances (Wang and Feng, 2012), satellite elevations ( $\mathrm{Li}$ et al., 2014), satellite variances ( $\mathrm{Li}$ and Teunissen, 2014), combined phase observation wavelengths ( $\mathrm{Li}$ et al., 2015, b) and composite methods that combine such strategies (Gao et al., 2017). In addition, the algorithm of satellite selection algorithm for PAR (Wang and Feng, 2013) and the method of EWL/WL as well as NL PAR for triple-frequency GNSS signals (Li et al., 2015a) are studied systematically. 
Many studies have applied the PAR method to the LC strategy and have achieved significant results. For example, the reliability characteristics of PAR solutions were verified by Wang and Feng (2012), and the PAR method was applied to the LC RTK positioning with the GPS constellation and virtual Galileo constellation to demonstrate the advantages of the proposed PAR method. Hou and Verhagen (2014) proposed a model and data driven PAR (MD-PAR) strategy and evaluated the performance of MD-PAR for GPS + BDS LC RTK positioning using simulated GPS and BDS observations. $\mathrm{Li}$ et al. $(2015, \mathrm{~b})$ presented the multi-carrier fast PAR (MCFPAR) strategy to solve multi-system multifrequency high-dimensional AR problems, and its validity was demonstrated with BDS + GPS LC RTK positioning using real dual- and triple-frequency observations. Gao et al. $(2015,2015)$ quoted the partial widelane ambiguity resolution strategy to GPS + BDS LC RTK positioning and GPS+ GLONASS + BDS LC RTK positioning and validated with real observations.

However, there are few publications applying the PAR method to the TC strategy, although this strategy provides more observations. The PAR method was introduced into the GPS + Galileo TC RTK positioning by Cao et al. (2007), and verify the reliability performance of this strategy in short baseline RTK. However, the simulation data is used and the intersystem bias is ignored.

In this paper, a GPS + Galileo TC RTK positioning strategy with PAR method is proposed. A set of real baseline observations ranging from about 22 to $110 \mathrm{~km}$ are used to test the performance of this strategy, including success rate, convergence time and ratio values. The experimental results are provided to demonstrate the benefits of introducing the PAR method into the TC strategy for multi-GNSS, which is finally followed by the summary and conclusions of this study.

\section{Methods}

\section{Multi-GNSS observation models}

Without loss of simplicity, the DD pseudorange and carrier-phase observation equations can be expressed as

$$
\begin{aligned}
& P_{r_{1} r_{2}, i j}^{S_{1} s_{2}, A_{1} A_{2}}=\rho_{r_{1} r_{2}}^{s_{1} s_{2}}+u c d_{r_{1} r_{2}, i j}^{A_{1} A_{2}}+I_{r_{1} r_{2}, i j}^{s_{1} s_{2}}+T_{r_{1} r_{2}}^{S_{1} s_{2}}+\varepsilon_{P_{r_{1}} s_{2}}^{s_{1} s_{2}} \\
& \Phi_{r_{1} r_{2}, i j}^{s_{1} s_{2}, A_{1} A_{2}}=\rho_{r_{1} r_{2}}^{s_{1} s_{2}}+u p d_{r_{1} r_{2}, i j}^{A_{1} A_{2}}-I_{r_{1} r_{2}, i j}^{S_{1} s_{2}}+T_{r_{1} r_{2}}^{S_{1} s_{2}} \\
& +\lambda_{i} N_{r_{1} r_{2}, i}^{s_{1}}-\lambda_{j} N_{r_{1} r_{2}, i}^{s_{2}}+\varepsilon_{\Phi_{r_{1} r_{2}}^{s_{2} s_{2}}}
\end{aligned}
$$

where $P$ and $\Phi$ are pseudorange and carrier phase measurements, respectively; $\rho$ is the distance between the receiver and the satellite; ucd and upd are receiver uncalibrated code and phase delays, respectively; These two quantities are related to the initial phase and the hardware phase delays (Gu, 2013); The symbol $I$ denotes the ionospheric delay; $T$ is the tropospheric delay; $\lambda$ is the wavelength; $N$ is the integer phase ambiguity; $\varepsilon_{P}$ and $\varepsilon_{\Phi}$ are the mixture of measurement noise and multipath error for pseudorange and carrier phase observations, respectively. Note that all variables are expressed in meters, except the ambiguity which is expressed in cycles. Furthermore, the reference receiver is denoted with subscript $r_{1}$, the rover receiver is denoted using subscript $r_{2}$ , the reference satellite is denoted using superscript $s_{1}$ and its system is labeled using superscript $A_{1}$, the nonreference satellite is denoted using superscript $s_{2}$ and its system is labeled using superscript $A_{2}$, and superscript $i$ and $j$ refer to carrier frequencies.

The above observation equations for multi-GNSS DD operations can be generalized to inter-system mixed DD which can be further categorized into those between the same frequencies or the diverse frequencies of observations (Li et al., 2017).

Since GPS and Galileo transfer the same frequency band signals, e.g., L1 and L5 signals respectively overlap E1 and E5a signals, the inter-system mixed DD model for the same frequency is used to realize the tight combination of GPS and Galileo measurements which can be expressed as follows

$$
\begin{gathered}
P_{r_{1} r_{2}, i}^{s_{1} s_{2}, A_{1} A_{2}}=\rho_{r_{1} r_{2}}^{s_{1} s_{2}}+u c d_{r_{1} r_{2}, i}^{A_{1} A_{2}}+I_{r_{1} r_{2}, i}^{s_{1} s_{2}}+T_{r_{1} r_{2}}^{s_{1} s_{2}} \\
+\varepsilon_{P_{r_{1} r_{2}}^{s_{1} s_{2}}} \\
\Phi_{r_{1} r_{2}, i}^{s_{1} s_{2}, A_{1} A_{2}}=\rho_{r_{1} r_{2}}^{s_{1} s_{2}}+u p d_{r_{1} r_{2}, i}^{A_{1} A_{2}}-I_{r_{1} r_{2}, i}^{s_{1} s_{2}}+T_{r_{1} r_{2}}^{s_{1} s_{2}} \\
+\lambda_{i} N_{r_{1} r_{2}, i}^{s_{1} s_{2}}+\varepsilon_{\Phi_{r_{1} r_{2}}^{s_{1} s_{2}}}
\end{gathered}
$$

Because the frequencies are the same, the ambiguities $N_{r_{1} r_{2}, i}^{s_{1} s_{2}}$ still have integer characteristics. However, the receiver UPDs which are related to the initial phase and hardware delay are consequently contained in the intersystem bias (ISB) and therefore cannot be eliminated, i.e. $u c d_{r_{1} r_{2}, i}^{A_{1} A_{2}} \neq 0$ and $u p d_{r_{1} r_{2}, i}^{A_{1} A_{2}} \neq 0$.

The carrier phase integer ambiguities $N_{r_{1} r_{2}, i}^{s_{1} s_{2}}$ and the integer part of $u p d_{r_{1} r_{2}, i}^{A_{1} A_{2}}$ are linearly dependent which make it impossible to separate them in the least-squares adjustment due to rank deficiency. Here, we separate the $u p d_{r_{1} r_{2}, i}^{A_{1} A_{2}}$ into a fractional part $\overline{u p d_{r_{1} r_{2}, i}^{A_{1} A_{2}}}$ and an integer part. Then, the remaining integer part $M_{r_{1} r_{2}, i}^{A_{1} A_{2}}$ is combined with the integer ambiguities $N_{r_{1} r_{2}, i}^{s_{1} s_{2}}$ and forms a new estimable integer parameter: $\overline{N_{r_{1} r_{2}, i}^{s_{1} s_{2}}}=N_{r_{1} r_{2}, i}^{s_{1} s_{2}}$ $+M_{r_{1} r_{2}, i}^{A_{1} A_{2}}$. Consequently, the Eq. (4) can be rewritten as 


$$
\begin{gathered}
\Phi_{r_{1} r_{2}, i}^{s_{1} s_{2}, A_{1} A_{2}}=\rho_{r_{1} r_{2}}^{s_{1} s_{2}}-I_{r_{1} r_{2}, i}^{s_{1} s_{2}}+T_{r_{1} r_{2}}^{s_{1} s_{2}}+\lambda_{i} \overline{N_{r_{1} r_{2}, i}^{S_{1} s_{2}}} \\
\quad+\overline{u p d_{r_{1} r_{2}, i}^{A_{1} A_{2}}}+\varepsilon_{\Phi_{r_{1} r_{2}}^{s_{1} s_{2}}}
\end{gathered}
$$

The carrier phase and code ISBs between different types of receivers have temporal stability and can be neglected between receivers of the same type. Therefore, if the ISB of a pair of receivers is estimated, it can be used as the ISB correction for the pair of receivers (Paziewski and Wielgosz, 2015). The phase and code ISBs can be estimated precisely for zero or ultra-short baselines, that is

$$
\left\{\begin{array}{l}
u c d_{r_{1} r_{2}, i 0}^{A_{1} A_{2}}=P_{r_{1} r_{2}, i}^{s_{1} s_{2}, A_{1} A_{2}}-\rho_{r_{1}}^{s_{1} s_{2}} r_{2} \\
\overline{u p d_{r_{1} r_{2}, i \boldsymbol{O}}^{A_{1} A_{2}}}=\left(\Phi_{r_{1} r_{2}, i}^{s_{1} s_{2}, A_{1} A_{2}}-\rho_{r_{1} r_{2}}^{s_{1} s_{2}}\right) / \lambda_{i}-\left[\left(\Phi_{r_{1} r_{2}, i}^{s_{1} s_{2}, A_{1} A_{2}}-\rho_{r_{1} r_{2}}^{s_{1} s_{2}}\right) / \lambda_{i}\right]
\end{array}\right.
$$

where the function $[\cdot]$ is a rounding function.

Through the introduction of the above corrections, the inter-system mixed DD on the same frequency can be translated into system-specific DD models:

$$
\begin{aligned}
& \overline{P_{r_{1} r_{2}, i}^{s_{1} s_{2}, A_{1} A_{2}}}=P_{r_{1} r_{2}, i}^{s_{1} s_{2}, A_{1} A_{2}}-u c d_{r_{1} r_{2}, i 0}^{A_{1} A_{2}} \\
& =\rho_{r_{1} r_{2}}^{s_{1} s_{2}}+I_{r_{1} r_{2}, i}^{s_{1} s_{2}}+T_{r_{1} r_{2}}^{s_{1} s_{2}}+\varepsilon_{P_{r_{1} r_{2}}^{s_{1} s_{2}}} \\
& \overline{\Phi_{r_{1} r_{2}, i}^{s_{1} s_{2}, A_{1} A_{2}}}=\Phi_{r_{1} r_{2}, i}^{s_{1} s_{2}, A_{1} A_{2}}-\overline{u p d_{r_{1} r_{2}, i O}^{A_{1} A_{2}}} \\
& =\rho_{r_{1} r_{2}}^{s_{1} s_{2}}-I_{r_{1} r_{2}, i}^{s_{1} s_{2}}+T_{r_{1} r_{2}}^{s_{1} s_{2}}+\lambda_{i} \overline{N_{r_{1} r_{2}, i}^{s_{1} s_{2}}}+\varepsilon_{\Phi_{r_{1} r_{2}}^{s_{1} s_{2}}}
\end{aligned}
$$

which is the model used in this study to implement GPS + Galileo DD data processing.

\section{Ambiguity resolution in the DD model}

The GNSS linear observation equations of Eqs. (7) and (8) can be expressed as:

$$
\mathbf{y}=\mathbf{A x}+\mathbf{B N}+\boldsymbol{\varepsilon}
$$

where $\mathbf{y}$ is the vector of 'observed minus computed' DD observations; $\boldsymbol{x}$ is the vector of incremental baseline coordinates, the residual tropospheric zenith delay, and the DD ionospheric slant delays for each measurement epoch; $\mathbf{N}$ is the vector of carrier-phase integer ambiguities; $\boldsymbol{\varepsilon}$ is the vector of unmodeled effects and measurement noise. The matrices $\boldsymbol{A}$ and $\boldsymbol{B}$ are the corresponding design matrices of $\boldsymbol{x}$ and $\mathbf{N}$, respectively.

The float solution $\mathbf{X}$ and variance-covariance matrix $\mathbf{Q}$ from a least-squares estimation can be expressed as

$$
X=\left[\begin{array}{c}
\hat{x} \\
\hat{N}
\end{array}\right], Q=\left[\begin{array}{cc}
Q_{\hat{x}} & Q_{\hat{x} \hat{N}} \\
Q_{\hat{N} \hat{x}} & Q_{\hat{N}}
\end{array}\right]
$$

In these formulas, the integer ambiguity vector $\hat{N}$ is obtained by solving an ILS (integer least square) problem expressed as:

$$
\breve{N}=\underset{\boldsymbol{N} \in \boldsymbol{Z}}{\operatorname{argmin}}\left\{(\boldsymbol{N}-\hat{\boldsymbol{N}})^{T} \boldsymbol{Q}_{\hat{\boldsymbol{N}}}{ }^{-1}(\boldsymbol{N}-\hat{\boldsymbol{N}})\right\}
$$

To solve the ILS problem, the well-known LAMBDA (Teunissen, 1995) method and its extension MLAMBDA (Chang et al., 2005) are employed in this paper. The integer vector solution is validated using the following "Ratio-Test".

$$
\boldsymbol{R}=\frac{\left(\breve{N}_{2}-\hat{\boldsymbol{N}}\right)^{T} \boldsymbol{Q}_{\hat{N}}{ }^{-1}\left(\hat{\boldsymbol{N}}_{2}-\hat{\boldsymbol{N}}\right)}{(\breve{N}-\hat{\boldsymbol{N}})^{T} \boldsymbol{Q}_{\hat{N}}{ }^{-1}(\breve{N}-\hat{N})}>\boldsymbol{R}_{\text {thres }}
$$

where the ratio-factor $R$, defined as the ratio of the weighted sum of the squared residuals by the second best solution $\widetilde{\mathbf{N}}_{2}$ to the best $\widetilde{\mathbf{N}}$ is used to check the reliability of AR. In general, validation threshold $R_{\text {thres }}$ can be 1.5 to 3.0 (Wang and Feng, 2012), and we used 3.0 for this study.

After the validation, the remaining real-valued parameter estimates $\breve{\mathbf{x}}$ and the corresponding variancecovariance matrix $\mathbf{Q}_{\widetilde{\mathbf{x}}}$ can be updated by solving the following equations.

$$
\left\{\begin{array}{c}
\breve{x}=\hat{x}-Q_{\hat{x} \hat{N}} Q_{\hat{N}}{ }^{-1}(\hat{N}-\breve{N}) \\
Q_{x}=Q_{\hat{x}}-Q_{\hat{x} \hat{N}} Q_{\hat{N}}^{-1} Q_{\hat{N} \hat{x}}
\end{array}\right.
$$

If the validation fails, the current epoch will keep the ambiguities float instead.

\section{Partial ambiguity resolution strategy}

If integer ambiguities of all satellites are difficult to fix with the LAMBDA method, the partial ambiguity fixing will be considered. Then the ambiguity vector $\hat{N}$ is divided into two parts, and the corresponding variancecovariance matrix of the two parts

$$
\hat{\boldsymbol{N}}=\left[\begin{array}{c}
\hat{\boldsymbol{N}}_{a} \\
\hat{\boldsymbol{N}}_{b}
\end{array}\right], \mathrm{Q}_{\hat{\boldsymbol{N}}}=\left[\begin{array}{cc}
\mathrm{Q}_{\hat{\boldsymbol{N}}_{a}} & \mathrm{Q}_{\hat{\boldsymbol{N}}_{a} \hat{\boldsymbol{N}}_{b}} \\
\mathrm{Q}_{\hat{\boldsymbol{N}}_{b} \hat{\boldsymbol{N}}_{a}} & \mathrm{Q}_{\hat{\boldsymbol{N}}_{b}}
\end{array}\right]
$$

where $\hat{N}_{a}$ is a set of to-be-fixed ambiguities, and $N_{b}$ the remaining ambiguities.

If $\hat{\boldsymbol{N}}_{a}$ can be fixed reliably, similar to the real-valued parameter update process, the remaining ambiguities $\boldsymbol{N}_{b}$ and their variance-covariance matrix $\mathbf{Q}_{\hat{\mathbf{N}}_{b}}$ can be corrected with the fixed ambiguities:

$$
\left\{\begin{array}{l}
\tilde{\mathrm{N}}_{b}=\hat{\mathrm{N}}_{b}-\mathrm{Q}_{\mathbf{N}_{b} \mathbf{N}_{a}} \mathrm{Q}_{\mathbf{N}_{a}}^{-1}\left(\hat{\mathrm{N}}_{a}-\breve{\mathrm{N}}_{a}\right) \\
\mathrm{Q}_{\tilde{\mathbf{N}}_{b}}=\mathrm{Q}_{\hat{\mathbf{N}}_{b}}-\mathrm{Q}_{\hat{\mathbf{N}}_{b} \hat{\mathbf{N}}_{a}} \mathrm{Q}_{\hat{\mathbf{N}}_{a}}^{-1} \mathrm{Q}_{\hat{\mathbf{N}}_{a} \hat{\mathbf{N}}_{b}}
\end{array}\right.
$$

Then the LAMBDA method is used to fix $\tilde{\boldsymbol{N}}_{b}$, and if $\tilde{\boldsymbol{N}}_{b}$ can be fixed, $\breve{\mathbf{N}}_{a}$ and $\tilde{\boldsymbol{N}}_{b}$ are used to update $\breve{\mathbf{x}}$ and $\mathbf{Q}_{\mathbf{X}}$. Otherwise, only $\widetilde{\mathbf{N}}_{a}$ is used to update $\breve{\mathbf{x}}$ and $\mathbf{Q}_{\breve{\mathbf{X}}}$. 
In this paper, a PAR procedure is used to determine the subset of ambiguities which in terms of the success rate and the ratio test (Wang and Feng, 2012). Figure 1 presents the flowchart of this procedure.

First, the PAR process starts with the decorrelation of the ambiguities and the diagonal elements of the decorrelated matrix are sorted in the ascending order. We can get the diagonal element set $D=\left\{d_{1}, d_{2}, \cdots d_{i}, \cdots, d_{n} \mid d_{1}<d_{2}\right.$ $\left.<\cdots d_{i}<\cdots<d_{n}\right\}$ after sorting by conditional variance. Then, by updating the traversal of $i=n$ to the minimum threshold $i=n_{0}$ and pick the subset $D=\left\{d_{1}, d_{2}, \cdots d_{i}\right\}$ and the corresponding ambiguity subset $\hat{\boldsymbol{N}}_{a}\left(D_{i}\right)$ and variancecovariance matrices $\mathrm{Q}_{\hat{\boldsymbol{N}}_{a}}\left(D_{i}\right)$. The minimum threshold $n_{0}$ is typically 6 to ensure the selected satellites are still sufficient to get reliable positioning results. Then, the LAMBDA method is applied in the ambiguity search process. If $P_{S} \geq P_{S 0}$ and $R>R_{\text {thres }}$, the fixed ambiguities can be considered to pass the acceptance test and be used into the following position calculation. Otherwise, we will update the subset and repeat the ambiguity search and test. If the number of selected ambiguities is less than $n_{0}$, the iteration will stop and only the float solutions are made available.

\section{Results and Discussion}

In order to analyze the effect of PAR on GPS + Galileo TC RTK positioning in the medium to long-baselines,

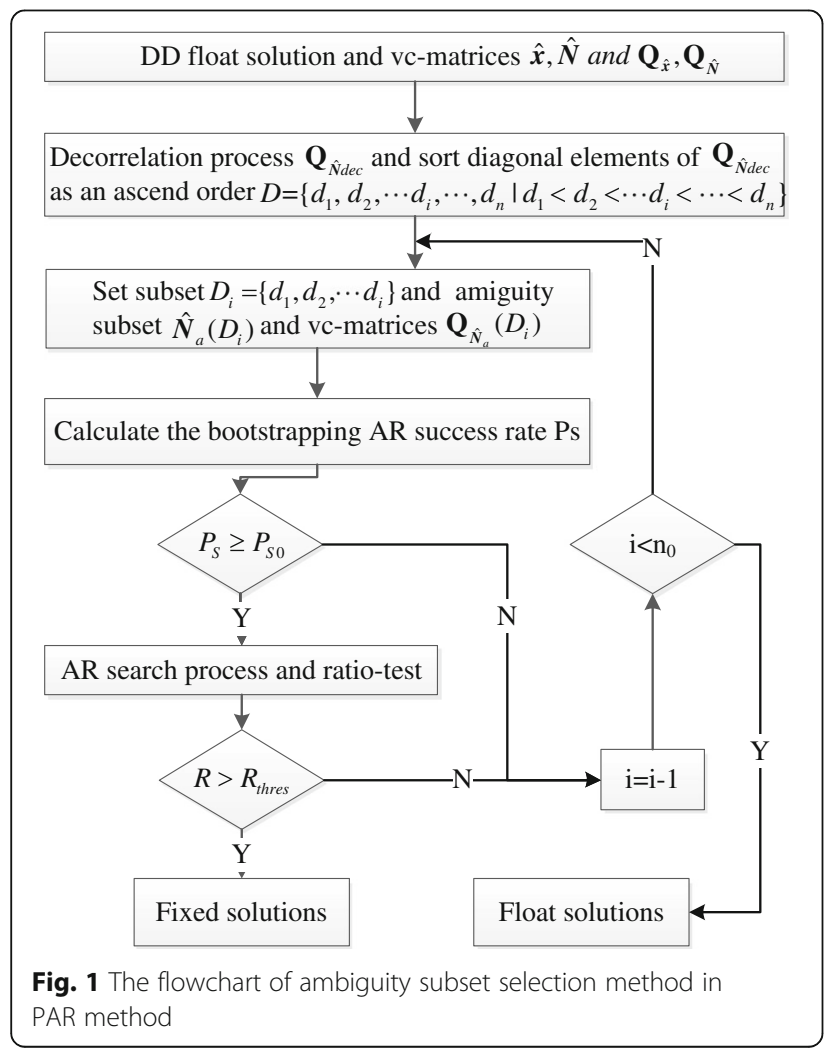

three stations with good observation conditions from the International GNSS Service (IGS) MGEX Network were selected for the experiments. The observation time covers 0:00:00 to 23:59:30 on July 7, 2017. The lengths of baselines and receiver type are given in Fig. 2.

The DD tropospheric and ionospheric delay could not be ignored in the medium-to-long baselines, and they would be set to zero initially and estimated as a random walk in each processing session in this paper. The processing settings for the relative positioning solutions of GPS + Galileo are given in Table 1.

\section{Carrier phase and code ISB estimation}

The estimates of phase and code ISBs can be accomplished on zero or short baselines, as described in the previous section. Here we used data from the GNSS Research Centre of Curtin University, with baseline information as showed in Table 2.

The fractional phase and code ISBs are calculated according to Eq. (6) and the results are shown in Figs. 3 and 4 Both figures show that the phase and code ISBs were stable during the period of daily experiments. However, there are differences in the ISBs of two groups of experiments over different periods. This is mainly due to the upgrade of the receiver firmware version, such as the CUT0 station's receiver firmware version from Trimble NETR9 (4.85) to Trimble NETR9 (5.20), which is similar to the results of (Paziewski and Wielgosz, 2015). Therefore, when estimating ISB, it is necessary to note not only the receiver type but also the receiver firmware version.

We selected the ISB corrections from the experiment in 2017 to correct the ISBs in the long-baseline experiment because the receiver's brands and firmware versions used in this experiment were the same as that used in the long-baseline experiment. The receiver brands and firmware versions of the long-baseline experiment are shown in Fig. 2.

\section{Results of ambiguity resolution}

The AR results of different combination strategy are shown for 500 min of data in Fig. 5 for the full ambiguity resolution (FAR) method and in Fig. 6 for the PAR method, including the AR success rate, number of ambiguities and ratio values. Note that in the ratio statistics, any ratio greater than 4 is assigned a value of 4 , which is intended to facilitate the distribution of smaller ratio values.

Figure 5 shows that the AR success rate and number of ambiguities of the three baselines are similar. However, the number of AR changes more frequently as the baseline length increases. When there is a newly-rising satellite, the success rate will drop dramatically. 


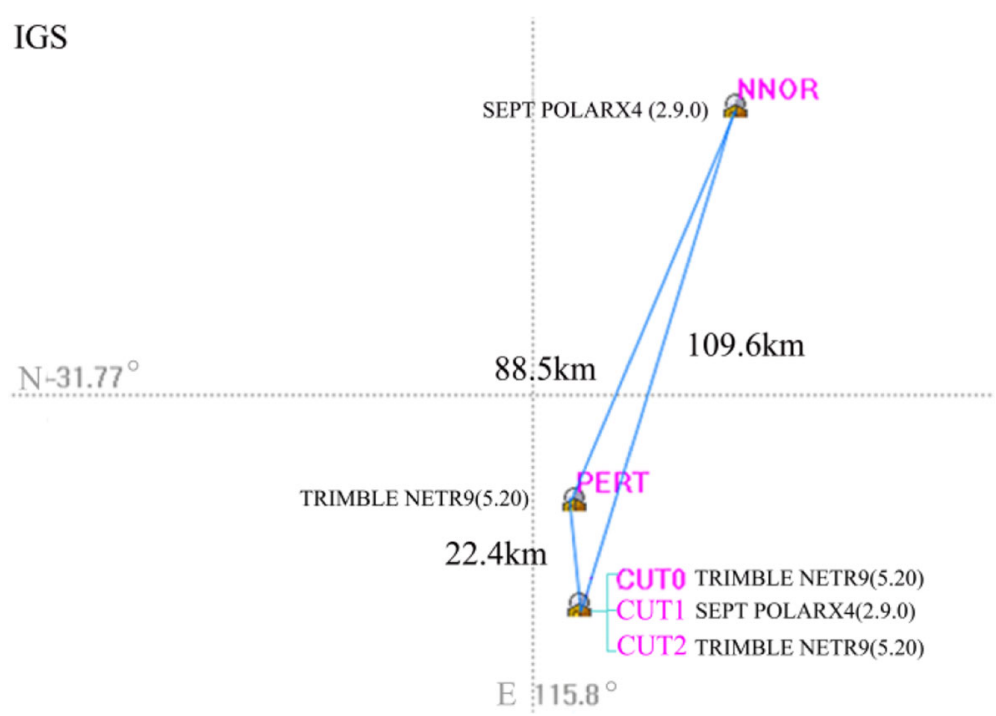

Fig. 2 Medium to long baselines used in the experiments

Fortunately, the TC strategy can provide more satellite observations, at least one more than the LC strategy. In this way, the TC strategy can be faster to reach the success rate at $99 \%$, and has the ability to eliminate the sudden change in the number of satellites.

Table 1 Medium and long-baseline data processing strategy settings

\begin{tabular}{ll}
\hline Item & Models \\
\hline Satellites & GPS + Galileo \\
Observations & Phase and code observations \\
Signal selection & GPS: L1, Galileo: E1 \\
Cutoff elevation & $15^{\circ}$ \\
Sampling rate & 30 s \\
Observation weight & Elevation dependent weight \\
Phase-windup effect & Corrected \\
Satellite Antenna PCO and PCV & DD elimination or weakening \\
Receiver antenna PCO and PCV & DD elimination or weakening \\
Earth tides & Corrected \\
Relativity correction & Corrected \\
Satellite orbit & Broadcast/Precise ephemerides \\
Satellite clock & DD elimination or weakening \\
Receiver clock & DD elimination or weakening \\
Station coordinate & estimated as parameters \\
Tropospheric delay & estimated as parameters \\
lonospheric delay & estimated as parameters \\
Phase ambiguities & LAMBDA/MLAMBDA \\
AR success rate threshold $P_{S O}$ & 0.999 \\
Ratio threshold $R_{\text {thres }}$ & 3 \\
Minimum threshold $n_{0}$ & 6 \\
\hline
\end{tabular}

It is worth noting that the ratio values of the mediumlong-baseline and the long-baseline are very different. The ratio of the medium-long-baseline ratio values greater than 3 is about $90 \%$ and the long-baseline is just about $5 \%$. This reflects the fact that the long-baseline is affected by the atmospheric delay error and is difficult to satisfy the condition of correct AR. In addition, TC did not improve the ratio values, but deteriorate. For example, the ratio of the NNOR-CUT0 long-baseline to the ratio values greater than 3 under the three different combinations of $\mathrm{LC}, \mathrm{TC}$, and tight combination with ISB corrections (TC+ISB) is 4.83, 4.16 and $4.34 \%$, respectively. This indicates that more observations do not increase the ambiguity ratio values, but rather deteriorate.

Figure 6 shows that the application of the PAR method can significantly shorten the convergence time. The three baselines under different combinations can all reach the success rate of $99 \%$ within two or three epochs.

For medium-long-baselines, the number of resolved ambiguity is relatively stable and the problem of newlyrising or falling satellites is effectively suppressed. Because the ambiguity subset selection process can remove these satellites according to the variance of their ambiguity, and the ambiguity variance of such satellites is generally large. In the following epochs, their

Table 2 Zero-Baselines used in the experiment of ISB estimates

\begin{tabular}{lll}
\hline Number & Station name & Receiver type \\
\hline 1 & CUT0-CUT1 & Trimble NETR9-Septentrio POLARX4 \\
2 & CUT0-CUT2 & Trimble NETR9-Trimble NETR9 \\
3 & CUT1-CUT2 & Septentrio POLARX4-Trimble NETR9 \\
\hline
\end{tabular}




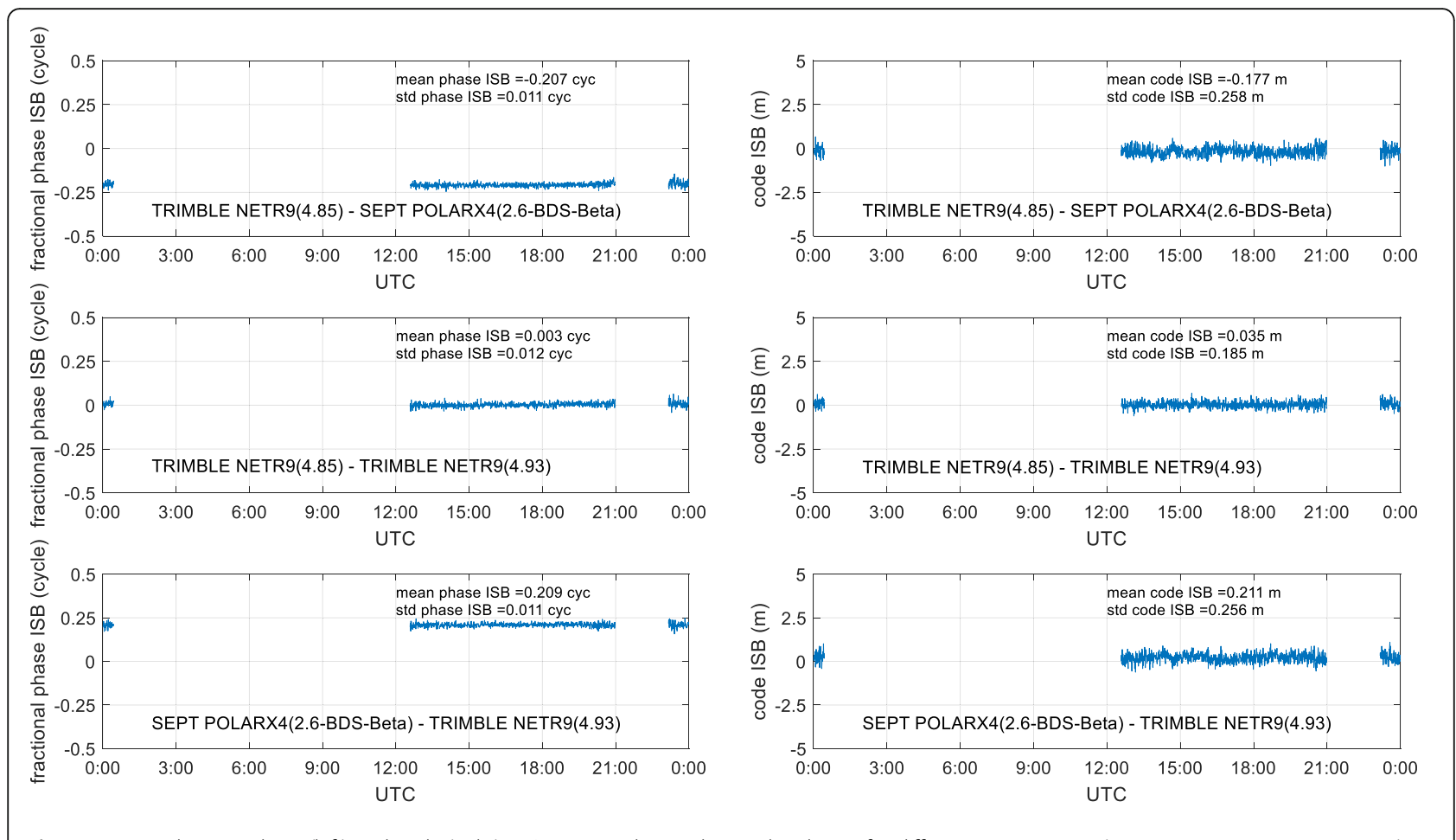

Fig. 3 Estimated carrier phase (left) and code (right) L1/E1 ISBs in the single-epoch solution for different receiver pairs (Experiment on January 25, 2015)

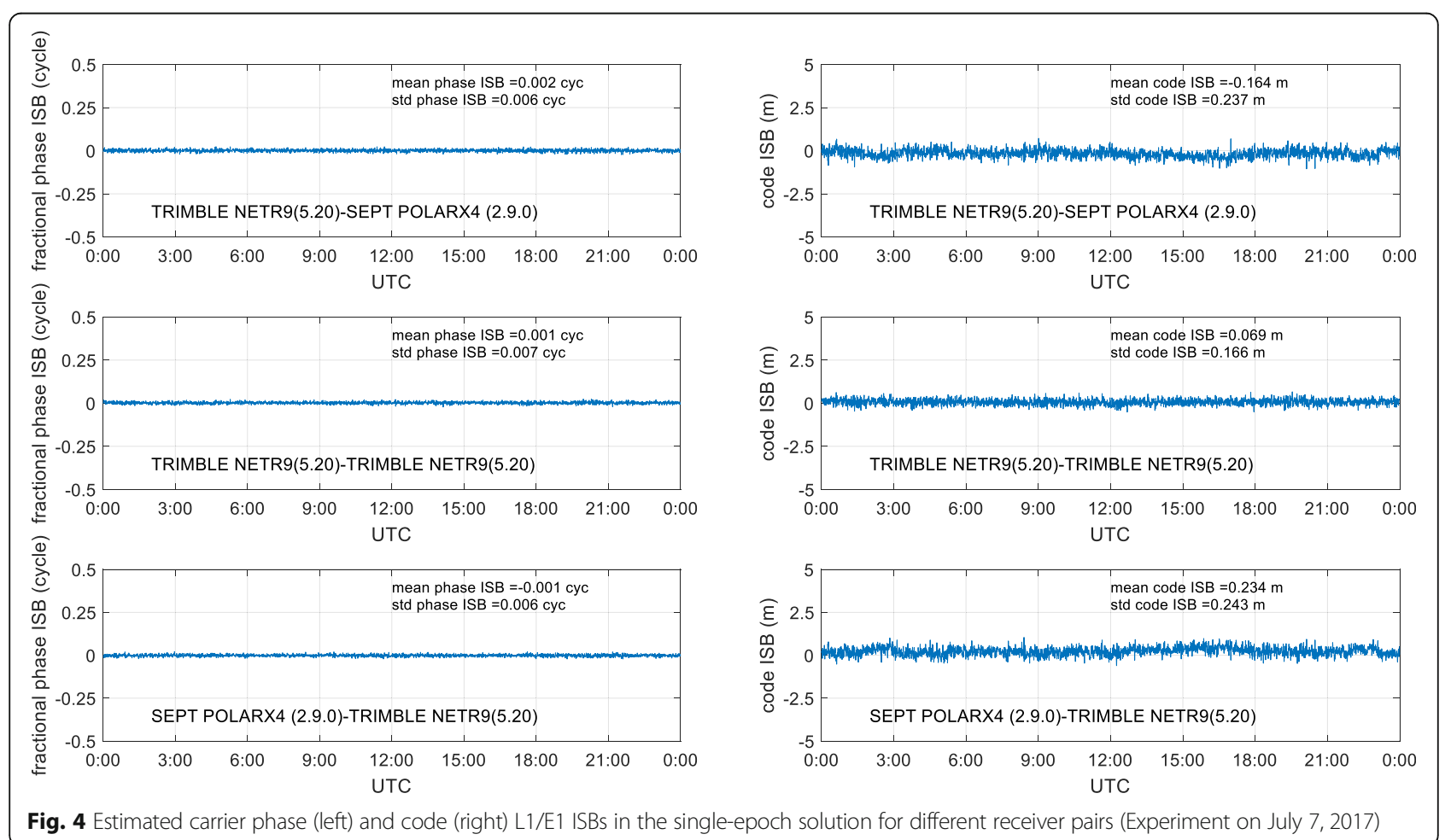




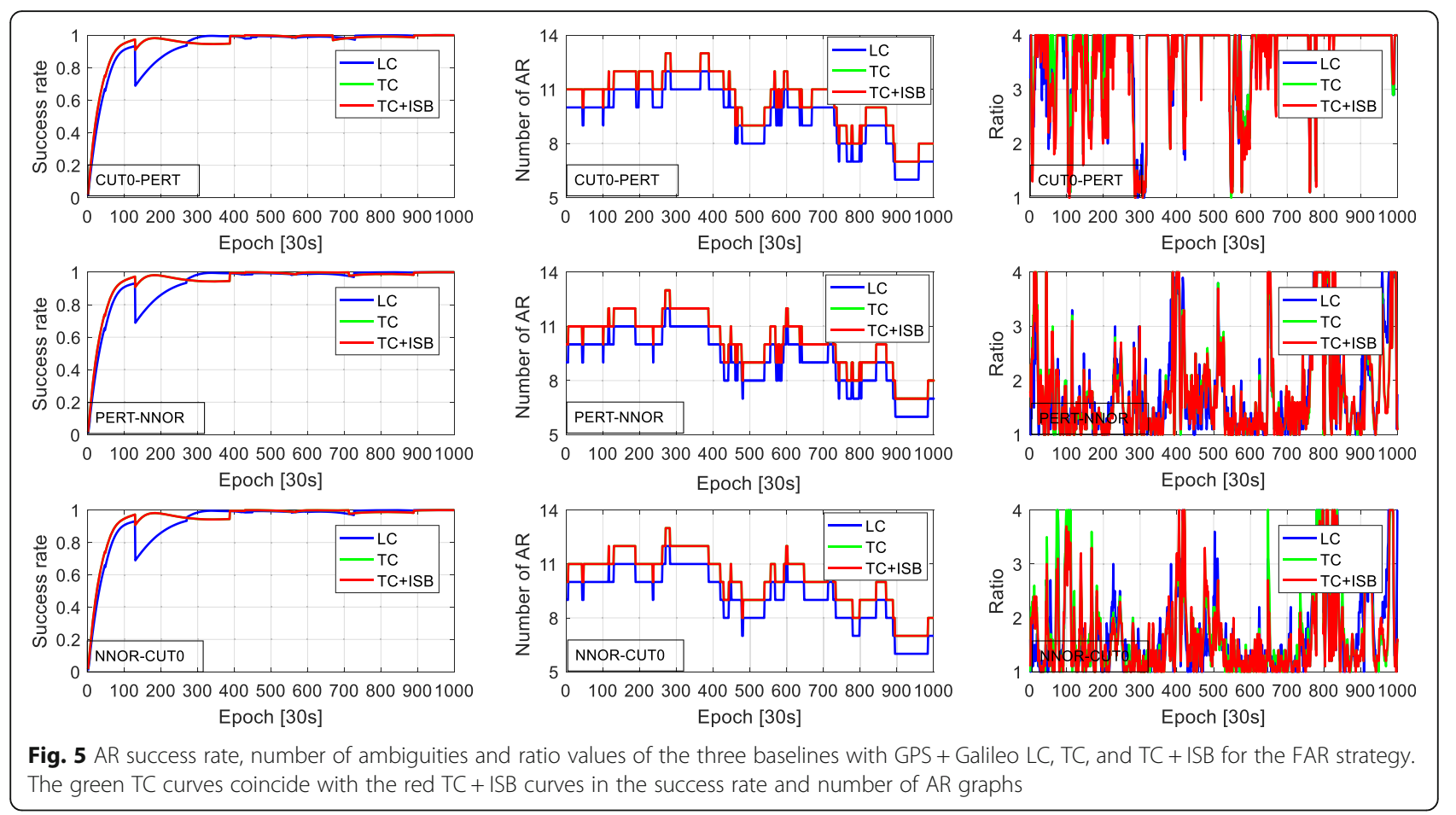

ambiguity precision improves, and their corresponding ratio values have also been improved, the ratio values also drops a lot only in the number of ambiguity changes epoch, but it still meets the threshold.

However, for long-baselines, the number of ambiguities to be fixed changes frequently. Because of the influence of atmospheric delay and so on, the ambiguity subset can't be fixed, even when the number of ambiguity reaches the threshold. Moreover, the frequent occurrence of falling satellites also affects the ambiguity subset fixed. Since the ambiguity subset can't be fixed, only the float solutions are available and then the

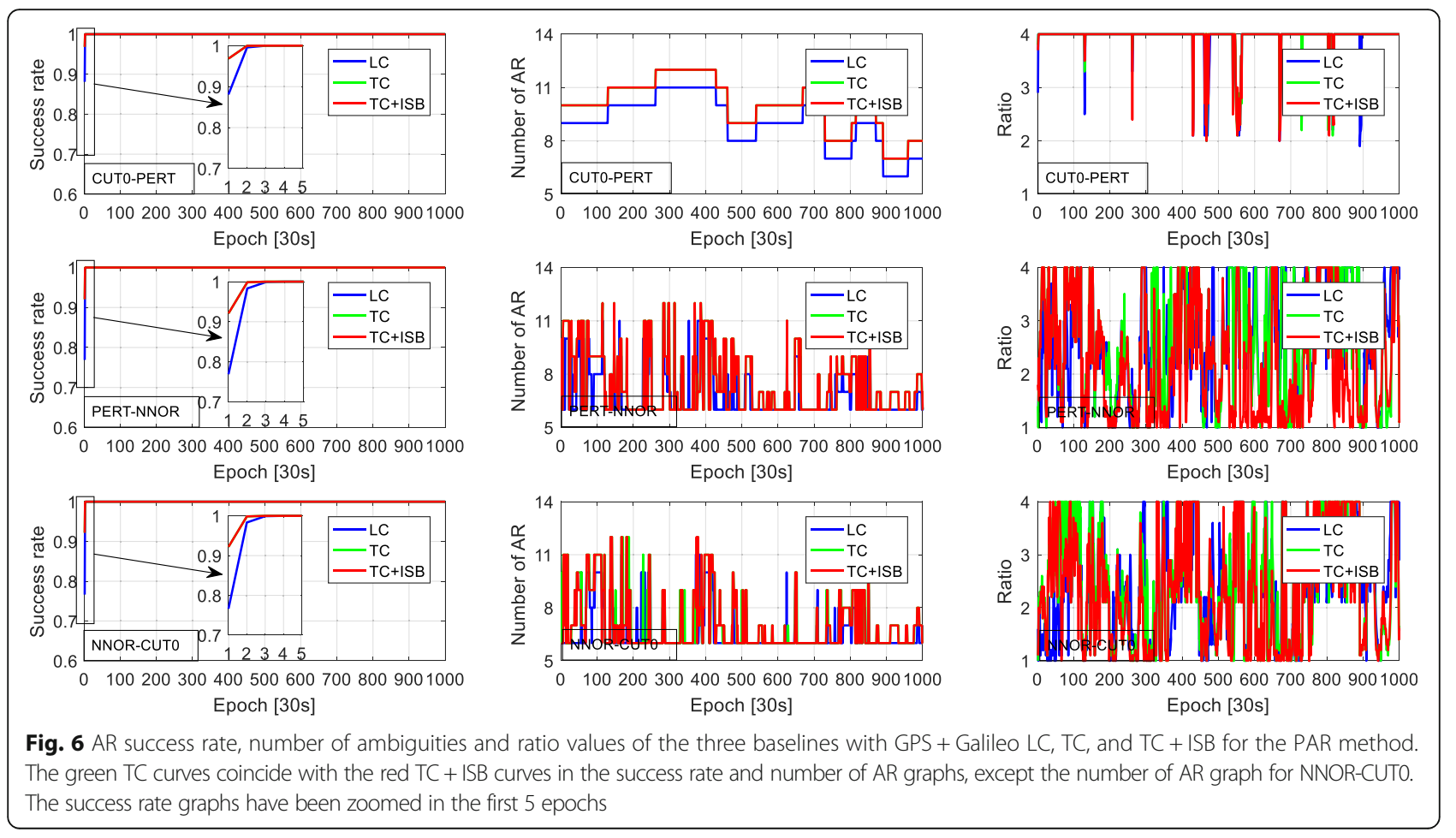


ambiguity subset selection is tried again in the next epoch. However, the PAR method is still able to greatly improve the ambiguity fixed rate. For example, the ratio of the NNOR-CUT0 long-baseline to the ratio values greater than 3 under the three different combinations of $\mathrm{LC}, \mathrm{TC}$, and TC+ISB for the PAR method is 27.36, 27.23 and $27.30 \%$, respectively. Compared to the FAR strategy, it was increased by $22.53,23.07$ and $22.96 \%$, respectively.

\section{Results of positioning}

The positioning capabilities of the two combination strategies and the two AR strategies are now verified with real GNSS data for single-frequency combined GPS + Galileo. The baseline errors of different combination strategies are shown in Figs. 7 and 8. Baseline errors are the difference between the estimated baseline length and precise reference baseline length.

Figure 7 shows that the medium-long-baseline error $(1.5 \mathrm{~cm})$ is smaller than that of the long-baseline $(10 \mathrm{~cm})$ static relative positioning for the FAR strategy. Affected by the residual atmospheric errors, such as residual tropospheric and ionospheric delays, the positioning results reflect some systematic biases especially for the longer baseline. The positioning accuracy based on the TC strategy is improved, especially for the medium length baseline. The accuracy of positioning based on TC + ISB is equivalent to that of TC, because the ISBs of this experiment are so small that it can be ignored. The positioning accuracy based on PAR method has improved, especially for the long-baseline. Because of after fixing the partial ambiguities by the PAR method, the fixed integer ambiguities can be back tracked into the observations to update the troposphere and the ionospheric parameters, a more accurate atmospheric delay correction is obtained, and the positioning parameters are updated by fixing the remaining ambiguities. The proportions of fixed solution of the NNORCUT0 long-baseline static relative positioning under the three different combinations of $\mathrm{LC}$, TC, and TC + ISB for the FAR method is $11.98,17.33$ and $17.40 \%$, respectively. For the PAR strategy, that is $75.80,76.70$ and $76.96 \%$, respectively.

Figure 8 shows that the baseline errors of different combination strategies RTK positioning for the FAR method and the PAR method. Introducing the PAR method into the TC strategy can not only fast converge, but also effectively reduce the baseline error. The results are similar to the static mode, however, the positioning accuracy and the proportion of the fixed solution decrease. For example, the proportions of fixed solution of the NNOR-CUT0 long-baseline RTK positioning under the three different combinations of $\mathrm{LC}, \mathrm{TC}$, and TC + ISB for the PAR method is 17.38, 18.23 and $18.69 \%$, respectively.

\section{Conclusions}

A GPS + Galileo tightly combined RTK positioning strategy is proposed for medium-to-long baselines, which introduces the PAR method to the strategy. The method
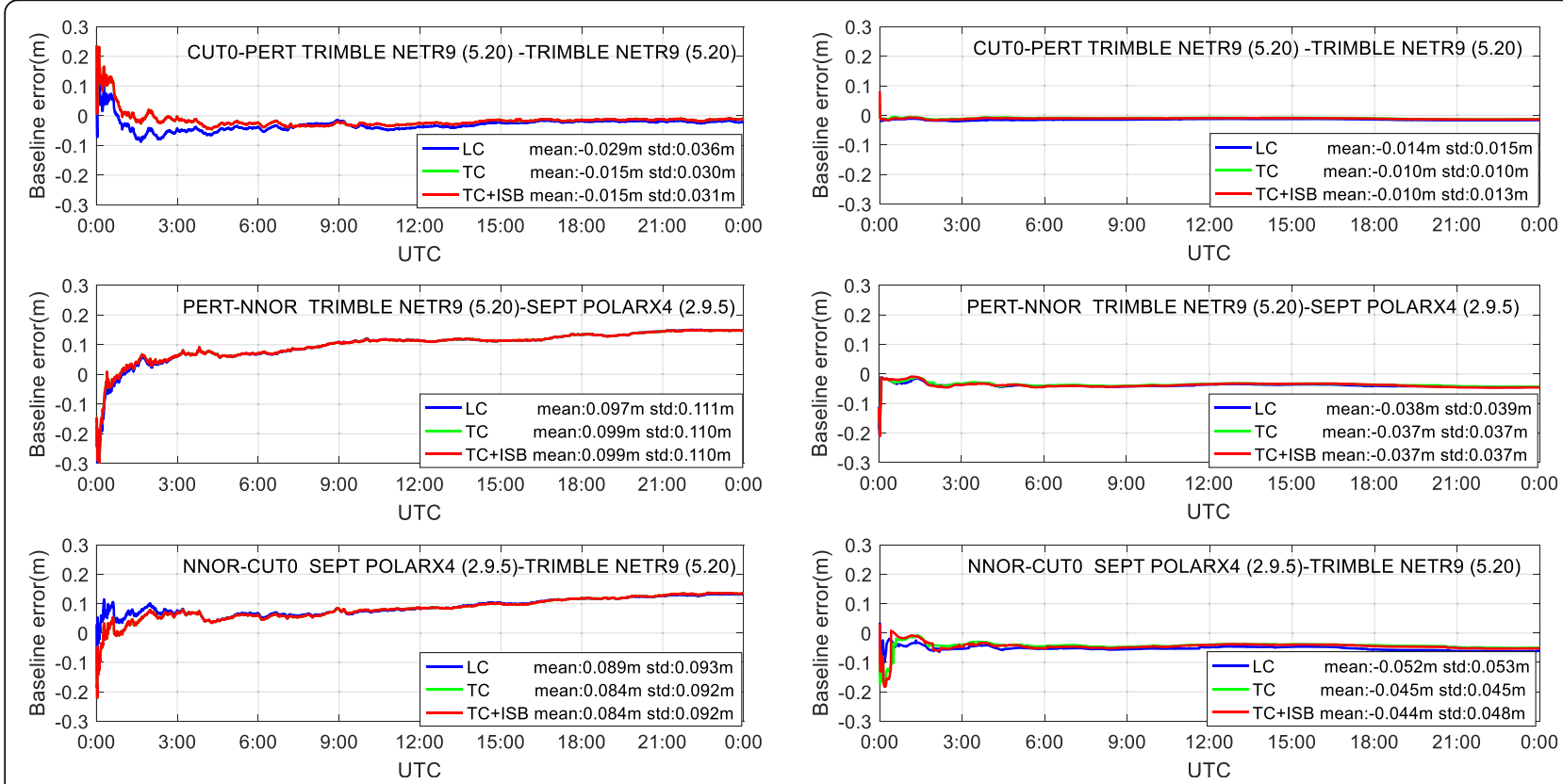

Fig. 7 Positioning results of the three baselines with GPS + Galileo LC, TC, and TC + ISB static relative positioning for the FAR method (left panel) and the PAR method (right panel). The green TC curves coincide with the red TC + ISB curves in the left panel 


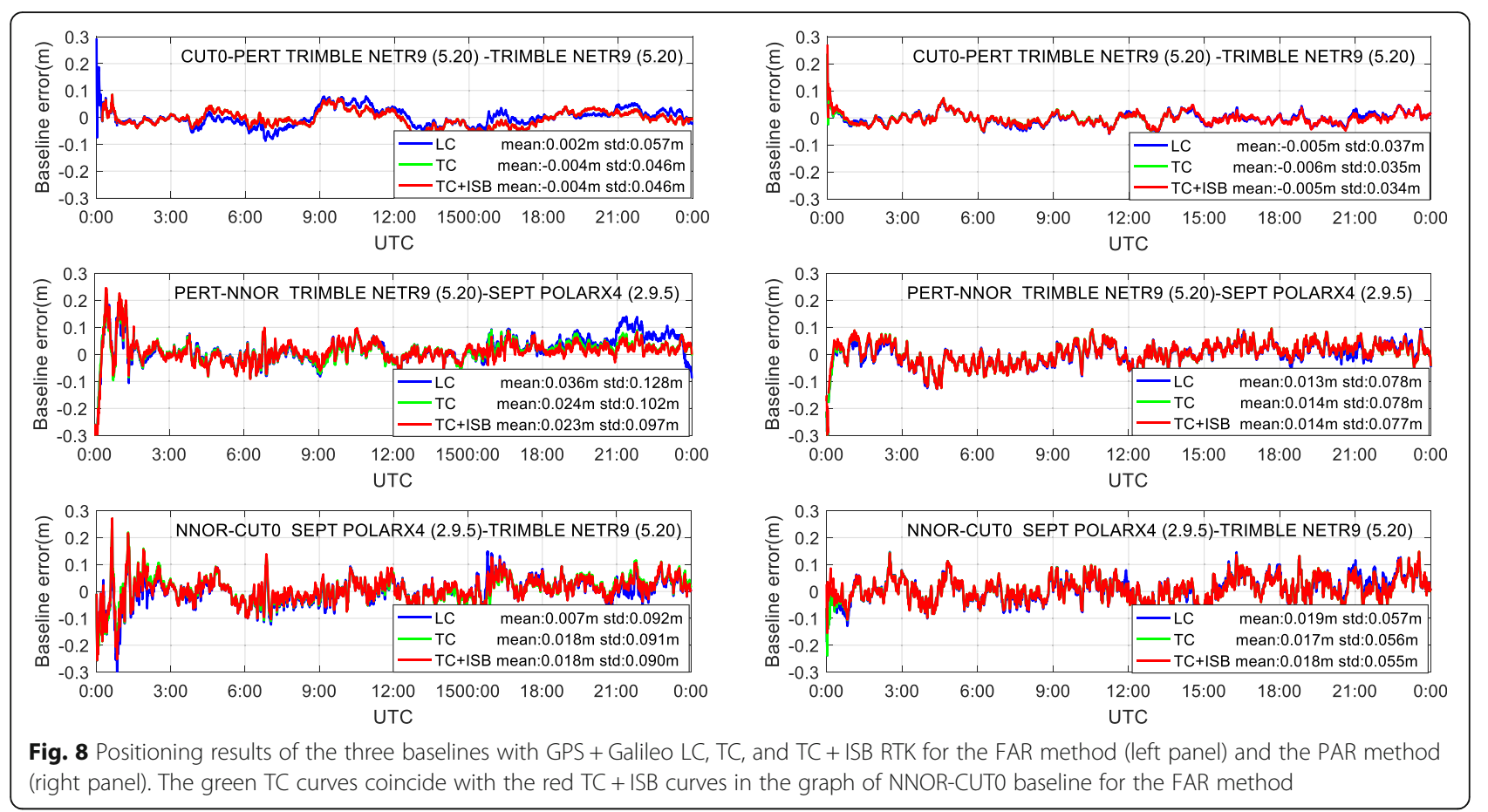

has been verified to be effective for faster and more reliable AR. Tests on middle-long and long-baselines demonstrate that TC strategy can provide more observations, which can improve the success rate. However, TC strategy does not increase the ambiguity ratio values, but rather deteriorate. The reason may be that the TC strategy increases the number of observations and increases the ambiguity dimension. Using the PAR method not only can make initialization time within three epochs, but also improve the ambiguity fixed rate. LC and TC strategies can get centimeter level positioning accuracy, but PAR of the TC strategy can provide better performance, especially for long-baselines. The selection of ambiguity subsets and the elimination of atmospheric delay are the areas that require further research in the future.

\section{Acknowledgments}

This work is funded by National Science Foundation of China (No.41674033) and State Key Research and Development Program (2016YFB0501802). We thank Curtin University for the baseline observations. We are also grateful for the high performance computing facility at Wuhan University which support all the computational work of this study.

\section{Authors' contributions}

$G L$ developed the algorithm. GL, JGeng and JGuo carried out most of the analyses and drafted the manuscript. SZ and SL participated in the design of the study and helped algorithm development. All authors have read and approved the final manuscript.

\section{Competing interests}

The authors declare that they have no competing interests.

\section{Publisher's Note}

Springer Nature remains neutral with regard to jurisdictional claims in published maps and institutional affiliations.

\section{Author details}

${ }^{1}$ GNSS Research Center, Wuhan University, Wuhan, China. ${ }^{2}$ Collaborative Innovation Center of Geospatial Technology, Wuhan, China. ${ }^{3}$ PowerChina Guiyang Engineering Corporation Limited, Guiyang, China. ${ }^{4}$ Chongqing Institute of Surveying and Mapping, NASG of china, Chongqing, China.

Received: 20 September 2017 Accepted: 23 January 2018

Published online: 02 March 2018

\section{References}

Cao W, O'Keefe K, Cannon ME (2007) Partial ambiguity fixing within multiple frequencies and systems, Proceedings of ION GNSS 2007. Fort Worth, TX, pp 312-323

Chang XW, Yang X, Zhou T (2005) MLAMBDA: a modified LAMBDA method for integer least-squares estimation. J GEODESY 79(9):552-565

Gao W, Gao C, Pan S (2017) A method of GPS/BDS/GLONASS combined RTK positioning for middle-long baseline with partial ambiguity resolution. Surv Rev 49(354):212-220

Gao W, Gao C, Pan S, Wang D, Deng J (2015) Improving ambiguity resolution for medium baselines using combined GPS and BDS dual/triple-frequency observations. SENSORS-BASEL 15(11):27525-27542

Gao W, Gao C, Pan S, Yang Y, Wang D (2015) Reliable RTK positioning method based on partial wide-lane ambiguity resolution from GPS/GLONASS/BDS combination, China satellite navigation conference (CSNC) 2015 proceedings: volume II. Springer, Berlin Heidelberg, pp 449-460

Gu S (2013) Research on the zero-difference un-combined data processing model for multi-frequency GNSS and its applications. Wuhan University, China, p 23

Hou Y, Verhagen S (2014) Model and data driven partial ambiguity resolution for multi-constellation GNSS, China satellite navigation Conference (CSNC) 2014 proceedings: volume II. Springer Berlin Heidelberg, pp 285-302

Julien O, Alves P, Cannon ME, ZHANG W (2003) A tightly coupled GPS/GALILEO combination for improved ambiguity resolution, Proc. ION GNSS 2003. European Navigation Conference, Graz, Austria, pp 1-14

Li B, Feng Y, Gao W, Li Z (2015) Real-time kinematic positioning over long baselines using triple-frequency BeiDou signals. IEEE Trans Aerospace Electron Syst 51(4):3254-3269

Li L, Li Z, Yuan H, Wang L, Hou Y (2016) Integrity monitoring-based ratio test for GNSS integer ambiguity validation. GPS SOLUT 20(3):573-585

Li B, Shen Y, Feng Y, Gao W, Yang L (2014) GNSS ambiguity resolution with controllable failure rate for long baseline network RTK. J GEODESY 88(2):99-112 
Li B, Teunissen PJG (2014) GNSS antenna array-aided CORS ambiguity resolution. J GEODESY 88(4):363-376

Li G, Wu J, Liu W, Zhao C (2016) A new approach of satellite selection for multiconstellation integrated navigation system, China satellite navigation conference (CSNC) 2016 proceedings: volume III. Springer, pp 359-371

Li G, Wu J, Zhao C, Tian Y (2017) Double differencing within GNSS constellations. GPS SOLUT 21(3):1161-1177. https://doi.org/10.1007/s10291-017-0599-4

Li J, Yang Y, Xu J, He H, Guo H (2015) GNSS multi-carrier fast partial ambiguity resolution strategy tested with real BDS/GPS dual- and triple-frequency observations. GPS SOLUT 19(1):5-13

Mowlam AP, Collier PA (2004) Fast ambiguity resolution performance using partially-fixed multi-GNSS phase observations. International symposium on GNSS/GPS, Sydney, Australia, pp 6-8

Paziewski J, Wielgosz P (2015) Accounting for Galileo-GPS inter-system biases in precise satellite positioning. J GEODESY 89(1):81-93

Teunissen PJ (1995) The least-squares ambiguity decorrelation adjustment: a method for fast GPS integer ambiquity estimation. J GEODESY 70(1):65-82

Teunissen P, Joosten P, Tiberius C (1999). Geometry-free ambiguity success rates in case of partial fixing. Proceedings of ION-NTM, pp. 25-27

Wang J, Feng Y (2012) Reliability of partial ambiguity fixing with multiple GNSS constellations. J GEODESY 87(1):1-14

Wang J, Feng Y. (2013). A satellite selection algorithm for achieving high reliability of ambiguity resolution with GPS and Beidou constellations. China satellite navigation conference (CSNC) 2013 proceedings. Springer Berlin Heidelberg, 2013: pp. 3-20

Zhang W, Cannon ME, Julien O, Alves P (2003) Investigation of combined GPS/ GALILEO cascading ambiguity resolution schemes, Proc. ION GPS/GNSS 2003 Portland, Oregon, pp 2599-2610

\section{Submit your manuscript to a SpringerOpen ${ }^{\circ}$ journal and benefit from:}

- Convenient online submission

- Rigorous peer review

- Open access: articles freely available online

- High visibility within the field

- Retaining the copyright to your article

Submit your next manuscript at $\gg$ springeropen.com 